\title{
The Al-Gazai-Donnai-Miller's syndrome as complication of the Hirsprung's disease has been always neglected. It is possible to cure it by the aids of cosmetic items
}

\section{Piotr Brzeziński', Lorenzo Martini ${ }^{1,2}$}

${ }^{1}$ University of Siena, Department of Pharmaceutical Biotechnologies, Via A. Moro 2, 53100 Siena, Italy, ${ }^{2}$ C.R.I.S.M.A. Inter

University Centre for Researched Advanced Medical Systems

Corresponding author: Prof. Lorenzo Martini, E-mail: martini36@unisi.it

Sir,

Hirschsprung disease-nail hypoplasia-dysmorphism syndrome is a fatal malformative disorder that is characterized by Hirschsprung disease, hypoplastic nails, albeit the real syndrome is called Al-GazaiDonnai-Miller's that involves distal limb hypoplasia and minor craniofacial dysmorphic features (flat facies, upward slanting palpebral fissures, narrow philtrum, narrow, high arched palate, micrognathia, low set ears with abnormal helices). Hydronephrosis has also been reported. There have been no further descriptions in the literature since 1988 [1].

The syndrome appears at the very first days of life and meanwhile the major part of complications (face dysfunctions) tend to disappear with the age, (bilateral hydronephrosis, imperforate anus was, obstructed bladder thought to be the result of posterior urethral valves, inguinal hernia) no sufficient attention has never been payed to hypoplastic nails (there are cases of people with six toes or lacking of a finger).

The AA of this modest case report focused their attention only to the hypoplasia of nails and not in children but in an old woman (66 y. old), who is diabetic.

Nails in diabetics must be always safe and neat and nail diseases are myriads [2].
In a male and female sib pair of Sikh origin and a male offspring of first-cousin Pakistani Muslims, Al-Gazali et al. described a combination of Hirschsprung disease (megacolon), hypoplastic nails, and minor dysmorphic facial features [3]. In one male infant, bilateral hydronephrosis was detected on routine ultrasound in midtrimester, and imperforate anus was noted at birth. Vesicostomy was performed for an obstructed bladder thought to be the result of posterior urethral valves. A left inguinal hernia was repaired during surgery for Hirschsprung disease. All 3 patients died as infants.

Dysmorphic facial features noted in the newborn period included low set, cup shaped ears with a thin outer helix, micrognathia, and a deep transverse groove on the chin. Other dysmorphic features included overlapping fingers which left hand of case 3 showing hypoplasia of distal were hypoplastic distally, hypoplastic finger and toe nails with an absent fourth left finger nail, and rocker bottom feet with an 'appended' fifth toe bilaterally. She developed bile stained vomiting on the second day and did not pass meconium.

In effect Nail dysplasia is often present at birth and can last all the life long and may present as nail hypoplasia, anonychia, longitudinal ridging, splitting, and spooning of the nail [4]. Triangular-appearing lunulae - the white areas at the base of the nail - are characteristic of NPS nail dysplasia [5]. Often the

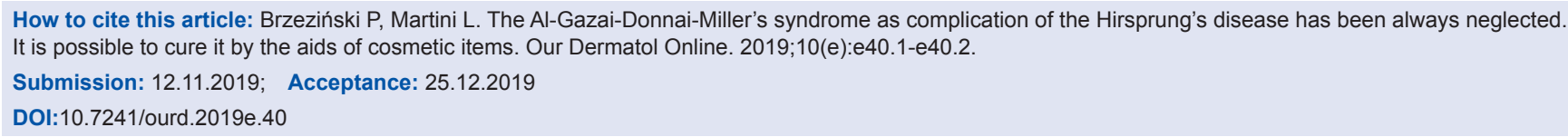


severity of the nail dysplasia increases as one progresses from the $5^{\text {th }}$ to the $1^{\text {st }}$ finger of the upper extremities. The nails of the lower extremity are less likely to be affected. Although nail dysplasia may present a cosmetic problem, it does not cause significant longterm morbidity.

For all these reasons the AA of this modest case report treated the concern from the cosmetological point of view, employing an hydroalcoholic nail enamel to be applied three times a day for 14 days.

This cosmetic system acts as a plastic layer and strengthen the same nail.

The mix was composed by: Ammonia; Sodium thiosulphate; Isopropyl alcohol; Water; Macadamia trinifolia seed oil

Our case shows the strengthening of the nail (especially the big toe) already at the $4^{\text {th }}$ day.

Remission is fully completed after 14 days of treatment.

\section{Consent}

The examination of the patient was conducted according to the Declaration of Helsinki principles.

\section{REFERENCES}

1. Davis C, Samarakkody U. Fryns syndrome: a surviving case with associated Hirschsprung's disease and hemidiaphragmatic agenesis. J Paediatr Child Health. 2002;38:318-20.

2. Omar J, Rajesh J. A survey of nail infection and awareness among non-diabetic patients in Mauritius. Our Dermatol Online. 2013;4:265-71.

3. Dunston JA, Lin S, Park JW, Malbroux M, McIntosh I. Phenotype severity and genetic variation at the disease locus: an investigation of nail dysplasia in the nail patella syndrome. Ann Hum Genet. 2005;69:1-8.

4. Stratigos AJ, Baden HP. Unraveling the molecular mechanisms of hair and nail genodermatoses. Arch Dermatol. 2001;137:1465-71.

5. Mellotte GJ, Eastwood JB. Pathognomonic sign of triangular lunulae in the nail-patella syndrome. Nephrol Dial Transplant. 1995;10:300-1-

Copyright by Piotr Brzeziński, et al. This is an open-access article distributed under the terms of the Creative Commons Attribution License, which permits unrestricted use, distribution, and reproduction in any

medium, provided the original author and source are credited.

Source of Support: Nil, Conflict of Interest: None declared. 